\section{Gender: missing the prizes that can inspire a career}

SIR - I congratulate Ben A. Barres on his excellent Commentary "Does gender matter?" (Nature 442, 133-136; 2006). I was struck by the paucity of female plenary lecturers at the Bioscience 2006 meeting of the UK Biochemical Society. Spurred on by Barres's comment that too few women academics speak out against prejudice, I decided to do a little research on the matter.

There have been three meetings of the Biochemical Society in the new annual meeting format (Biosciences 2004, 2005 and 2006) and at these 1 of 10,0 of 10 and 0 of 7, respectively, of the plenary lectures were given by a woman. Some of these plenary lecturers were recipients of prizes and medals, and I was so shocked by these statistics that I made a rough count of the proportion of women who have received these prizes over the years, as published on the society's website at www.biochemsoc.org.uk. Recipients' initials, rather than first names, are given, so I may conceivably have misattributed the male gender to some of the earlier names.

The prizes include the annual Colworth medal, given to a promising scientist under 35: only one has been awarded to a woman, out of 44 recipients, between 1963 and 2007. The statistics for the other prizes, up to 2007, are the Novartis medal, 2 of 39; Jubilee lecture, 1 of 23; Wellcome Trust award for research in biochemistry related to medicine, 1 of 11; AstraZeneca prize, 1 of 5; Frederick Gowland Hopkins memorial lecture, 0 of 24; Keilin memorial lecture, 0 of 21; Morton lecture, 0 of 14 ; Biochemical Society medal, 0 of 3 ; and GlaxoSmithKline medal, 0 of 2 . This translates into $3.2 \%$ of the prizes being given to women, a truly lamentable record.

Furthermore, the statistics have not improved. In the past ten years, none of the Colworth medals has been awarded to women - and it is prizes such as these, given to scientists early in their career, that influence their future success. The results speak for themselves: that people will always give prizes to others in their own image, unless forced to take sexual and racial bias into account. I wonder if the record of other scientific societies is much better in this regard.

I should also point out that UK Biochemical Society meetings are supported by funds from the Biotechnology and Biological Sciences Research Council and by the European Molecular Biology Organization. Why do research funding bodies not assert leverage on this matter, by insisting that sexual and racial bias in speaker selection must be addressed at any meeting for which their financial support is given?

\section{Annette C. Dolphin}

Laboratory of Cellular and Molecular

Neuroscience, Department of Pharmacology,

University College London, Gower Street,

London WC1E 6BT, UK

\section{Gender: macho language and other deterrents}

SIR - In the Commentary article "Does gender matter?" (Nature 442, 133-136; 2006), Ben A. Barres cites our article pointing out that the first round of the US National Institutes of Health (NIH) Pioneer awards was carried out in a way that would have predicted a bias against selection of women (M. Carnes et al. J. Womens Health 14, 684$691 ; 2005)$. Indeed, no women were selected in the first year, so when $43 \%$ of the second year's winners were women we examined the process again to see what had changed.

We identified several differences, including changes made by the NIH, that would predict a decrease in the activation of automatic gender stereotypes that may have discouraged women from applying and disadvantaged women applicants in the first round.

First, a reduction in the number of applicants (from 1,300 to 840) and greater familiarity with an application process that was nolonger new may have reduced time pressure on the reviewers.

Second, the NIH removed the repeated mention of the need for applicants to engage in 'high-risk' research; we believe that this terminology encouraged male and discouraged female applicants. Similarly, the emphasis on 'intrinsic' leadership abilities and 'potential" of the scientist was removed, in favour of an emphasis on the scientist's research.

Third, there was a much higher proportion of women in the applicant pool, which may have been related to the change in language (26\% in phase 1 and $35 \%$ in phase 2 in 2005 , compared with $20 \%$ and $10 \%$ in 2004). There was also a greater proportion of women on the review panel: $44 \%$ in 2005 , compared with $6 \%$ in 2004.

Fourth, the presence of accomplished women scientists on the review committee provided a positive role model for applicants.

Finally, women were specifically encouraged to apply - a particularly significant factor in the context of the outcry in the scientific community following the absence of women in the first round.

We applaud the NIH for taking an evidence-based approach. Regardless of the gender composition of the group selected in the forthooming third round, removal of conditions that are known to activate automatic gender stereotypes ensures that the best science will be supported, regardless of the sex of the scientist.

Molly Carnes

Department of Medicine, Psychiatry and Industrial \& Systems Engineering

University of Wisconsin-Madison, Madison, Wisconsin 53715, USA

See Nature 442, 510 (2006) for other letters on this topic. Readers are encouraged to add their comments on the Nature News Blog at: http://blogs.nature.com/news/ blog/2006/07/does_gender_matter.html

\section{A positive definition of prokaryotes}

SIR - In his Concepts essay, Norman R. Pace argues that the concept of prokaryotes is misleading and proposes that the word 'prokaryote' be banned from the scientific literature ${ }^{1}$. We disagree.

Pace contends that the term prokaryote refers to the lack of a nucleus and that it is hence a "negative and therefore scientifically invalid description" of cell organization, because "no one can define what is a prokaryote. The former is a matter of opinion, and the latter is arguably incorrect.

Prokaryotes are cells with co-transcriptional translation on their main chromosomes; they translate nascent messenger RNAs into protein. The presence of this character distinguishes them from cells that possess a nucleus and do not translate nascent transcripts on their main chromosomes ${ }^{2}$. Although historically founded on a negative trait (lacking a nucleus), the term prokaryote does indeed specifically designate organisms that are defined by a positive character.

Pace proposes that we should speak only of archaea and bacteria instead of prokaryotes, and that if a collective term is needed to designate those cells that are not eukaryotes, the term 'microbe' should be used. That suggestion, too, is unacceptable, because many eukaryotes are microbes.

Regardless of what any gene tree might suggest and regardless of what anyone might believe about early evolution, modern cells lacking spliceosomal introns and spliceosomes, a nucleus, and mitochondria ${ }^{3}$ do possess transcriptionally coupled translation - they are prokaryotes". William Martin ${ }^{\star}$, Eugene V. Koonin† *Institute of Botary, University of Dũsseldorf, 40225 Düsseldorf, Germany

†National Center for Biotechnology Information, National Library of Medicine, National Institutes of Health, Bethesda, Maryland 20894, USA

1. Pace, N. R. Nature 441,289 (2006)

2. Martin W.\& Koonin, E V. Nature 440, 41-45(2006).

3. Embley, T.M.\& Martin, W. Nature 440,623-630 (2006).

4. Wakh, D.A.\&Doolitte, W.F.Cur. Bbl. 15, R237-R240 (2005) 